\title{
Characterization of multi-layer porous media in an impedance tube
}

\author{
Rémi Roncen ${ }^{1}$ \\ ONERA/DMPE, Université de Toulouse, F-31055, Toulouse, France \\ Zine El Abiddinne Fellah ${ }^{2} \&$ Erick Ogam ${ }^{3}$ \\ Laboratoire de Mécanique et d'Acoustique, CNRS, UMR 7031, Aix-Marseille Université, Centrale \\ Marseille, F-13402 Marseille Cedex 20, France
}

\begin{abstract}
A general method is presented that allows for the inverse identification of the intrinsic properties of multi-layer porous materials in an impedance tube. To avoid the inverse problem ill-posedness, different air-gaps are placed behind the materials, artificially increasing the information contained within the observed signals. A Bayesian representation of knowledge is taken, where information on a material property is encoded in a probability density function. A numerical case with known material properties is shown as an example for the identification of a three-layer material.
\end{abstract}

\section{INTRODUCTION}

Porous materials are widespread elements that can be found in a wide variety of research areas such as geology, medicine and acoustics. The knowledge of their intrinsic properties (porosity, pore size, etc.) is a key element towards the proper characterization of their behaviour. Auspiciously, some of these intrinsic properties have been linked via semi-phenomenological models to the acoustic behaviour of porous materials. It thus stands to reason that inverse methods, based on acoustic measurements of a sound field interacting with a porous medium, can yield information on the pore micro-structure. This strategy is now routinely used in acoustic labs, often relying on the common impedance tube measurement to obtain the acoustic signals needed by the inverse method [1-6].

While the identification of a single-layer material has been broadly studied [7], there is very little in the literature about the identification of multi-layer or inhomogeneous materials with acoustic inverse methods $[6,8]$. When dealing with a fixed number of known materials that can be characterized independently, there is no need for such a multi-layer identification. However, when a single material is inhomogeneous and can be approximated by a discrete number of unknown layers, the method becomes relevant.

The main difficulty of acoustical inverse problems for parameter estimation is the ill-posedness of the problem, i.e., there is a non-uniqueness, an indetermination in the obtained solution $[2,9,10]$. The non-uniqueness of the solution can be partially characterized using a Bayesian inference approach, where the multi-modality of the parameters' pdf show each individual solution, and their likelihood.

\footnotetext{
${ }^{1}$ remi.roncen@onera.fr

${ }^{2}$ fellah@lma.cnrs-mrs.fr

${ }^{3}$ ogam@lma.cnrs-mrs.fr
} 
A more thorough numerical investigation on the topic is available in Ref. [11], where additional details can be found.

The models and methods used in the article are first recalled succinctly in Sec. 2. A common pitfall leading to biased results in inverse problems based on impedance tube measurements is highlighted in Sec. 3 , followed by the formulation of a straightforward solution. In addition, a simple measurement scheme is showed in Sec. 4 that can be used to remove some of the inverse problem ill-posedness. This technique is based on the addition of air-gaps behind the acoustic material. Conclusions are drawn in Sec. 5.

\section{MODELS AND METHODS}

The present section gives a brief account of the acoustic modelling that was used in this work, as well as the Bayesian inference approach that was followed to carry the parameter estimation.

\subsection{Acoustic modelling}

Only rigid porous media are considered in this work, assumed infinite in all but one dimension (the thickness is finite). A transfer matrix approach is followed to evaluate the normalized surface impedance $Z_{s}$ of a multi-layer material [12, Chap. 11], and thus its reflection coefficient $\tilde{R}$.

The pore dissipation model is the JCAPL model [13-16] coupled with the hypotheses on the pore distribution by Horoshenkov [17], i.e., the pore sizes follow a log-normal distribution and the pores have a circular shape. This specialized model is used in order to limit the number of parameters to estimate. The model parameters are the porosity $\phi$, the pore mean size $\bar{s}$ and the pore standard deviation $\sigma_{s}$.

Given a certain impedance tube (see Fig. 1 for a schematics), one can calculate the pressure field within the tube at at microphone locations in a direct way as

$$
p(x, \omega)=\tilde{A} e^{-\mathrm{j} k(\omega) x}+\tilde{A} \tilde{R}(\omega) e^{+\mathrm{j} k(\omega) x},
$$

with $\tilde{A}$ the source coefficient, $\mathrm{j}$ the imaginary unit, $x$ the longitudinal microphone locations in the tube and where $k(\omega)=\omega / c_{f}$ is the propagation wavenumber of air for the plane wave (here the tube is assumed wide enough to neglect viscous effects at the walls), with $c_{f}$ the speed of sound.

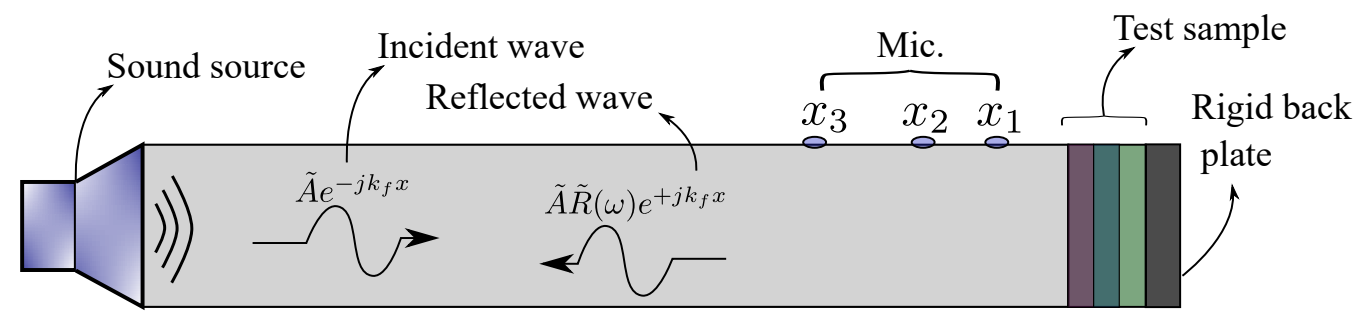

Figure 1: Schematics of an impedance tube.

These pressure fields, evaluated at 3 discrete locations to mimic an experiment with microphones, are used as inputs of the inverse problem instead of the impedance or the reflection coefficient. The main reason is to avoid a bias during the identification, as explained in Sec. 3.

\subsection{Bayesian Inference for Parameter Estimation}

Using Bayesian inference, one can interpret the degree of belief on estimated parameters via probabilities [18, Chap. 8]. This approach is an alternative to classical deterministic inverse problems, where a minimization is performed to match observed and modelled data. In the present study, we use a MCMC approach to approximate the pdfs of all the parameters of the problem. 
In particular, the strategy of Ref. [19] is used, as it allows for the identification of multimodal distributions even in a high dimension parameter space. This is particularly attractive since nonuniqueness of the solution is expected in the inverse problem at hand. The same strategy was applied for porous media parameter identification in Refs. $[9,10]$, albeit for single layer porous samples.

\section{AN AVOIDABLE SOURCE OF BIAS}

It may seem logical to the practitioner to use the impedance spectrum or the reflection coefficient spectrum, measured in an impedance tube, as the observable data used as inputs in the Bayesian inference process (or a deterministic approach) for parameter estimation. However, a given constant uncertainty on the measured pressure at the microphone location does not translate into a constant uncertainty in impedance of reflection coefficient.

A numerical example is given in Fig. 2, where the reflection coefficient is shown with credibility intervals. In this case, the numerical pressures are polluted with a Gaussian white noise whose amplitude is $5 \%$ of the incident wave amplitude $\tilde{A}$. A pseudo-inverse strategy is performed to identify the reflection coefficient, taking as inputs the pressure measurements. This is done $10^{4}$ times with different noises added to the "true" pressure measurements, to average the results. As seen in
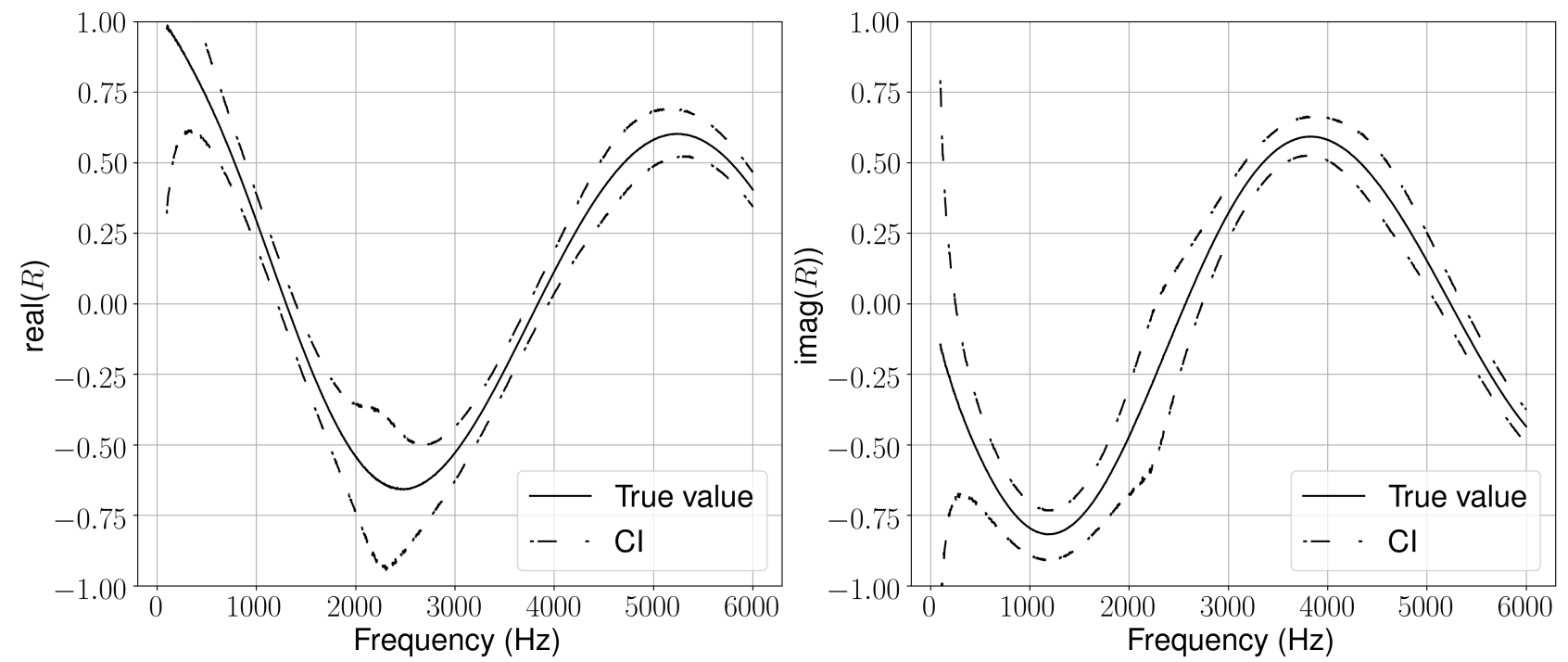

Figure 2: Real and imaginary parts of the reflection coefficient of a foam of thickness $30 \mathrm{~mm}$ with $\phi=0.98, \bar{s}=250 \mu \mathrm{m}$ and $\sigma_{s}=0.05$. The continuous line are the true values. The dashed lines correspond to the intervals containing $\approx 95 \%$ of the $10^{4}$ samples that were used to obtain converged statistics.

Fig. 2, the uncertainty is not constant anymore in the signal. It increases sharply at low frequency because of the pseudo-inverse matrix conditioning (relates to microphone spacing) and near $2 \mathrm{kHz}$ for an unknown reason. Since the uncertainty of the observed signal is directly responsible for the uncertainty on the estimated parameters, it stands to reason to use pressure measurements instead of signals derived from them (i.e., impedance or reflection coefficient). In the present method, a constant uncertainty is assumed on the pressure measurements (zero-mean Gaussian white noise of standard deviation $\sigma_{\mathfrak{R}}$ for the real part of the pressure and $\sigma_{\mathfrak{J}}$ for the imaginary part).

\section{MULTI-LAYER IDENTIFICATION}

Using numerical noisy measurements at three microphone locations as observed inputs, a Bayesian inference is performed using the MCMC technique of Ref. [19]. A serial assembly of three different 
foams is considered. Their properties are listed in Table. 1. Since the same model is used for generating the data and for estimating the parameters, an inverse crime is realised (despite the presence of noise in the input data). This problem will be addressed in a future work by considering different pore dissipation models. For the present study, we argue that the main goal of removing the inverse problem ill-posedness can still be well exemplified with an inverse crime, where the exact parameter values are known and can be used for comparison.

Table 1: True parameters of the samples, with $L$ the sample thickness, $\phi$ the porosity, $\bar{s}$ the pore mean size and $\sigma_{s}$ the pore standard deviation (in $\log 2$ base).

\begin{tabular}{ccccc}
\hline Material & $L(\mathrm{~mm})$ & $\phi$ & $\bar{s}(\mu \mathrm{m})$ & $\sigma_{s}$ \\
\hline$M_{1}$ & 10 & 0.85 & 100 & 0.2 \\
\hline$M_{2}$ & 10 & 0.65 & 50 & 0.5 \\
\hline$M_{3}$ & 10 & 0.98 & 250 & 0.05 \\
\hline
\end{tabular}

The prior knowledge is encoded in the Bayesian inference via uniform probabilities having a large support, to perform a blind inference where little information is given. The bounds of these supports are given in Table 2 . The true value of the ambient temperature is $20^{\circ} \mathrm{C}$, the true value of the ambient pressure is $10^{5} \mathrm{~Pa}$, the true value of the standard deviation on the real part of the pressure measurement is $\sigma_{\Re}=0.04 \mathrm{~Pa}$ and for the imaginary part, $\sigma_{\mathfrak{I}}=0.08 \mathrm{~Pa}$.

Table 2: Prior bounds of the parameters, where the symbol - means that there is no unit. The indices $i$ and $j$ denote the layer number and air-gap number, respectively. The $*$ exponent means the true value of a parameter.

\begin{tabular}{cccccccccc}
\hline Parameter & $\sigma_{\Re}$ & $\sigma_{\mathfrak{I}}$ & $T$ & $P_{0}$ & $L_{p, i}$ & $\phi_{i}$ & $\bar{s}_{i}$ & $\sigma_{s, i}$ & $L_{a, j}$ \\
\hline Unit & $\mathrm{Pa}$ & $\mathrm{Pa}$ & ${ }^{\circ} \mathrm{C}$ & $10^{5} \mathrm{~Pa}$ & $\mathrm{~mm}$ & - & $\mu \mathrm{m}$ & - & $\mathrm{mm}$ \\
\hline Min & $10^{-4}$ & $10^{-4}$ & 5 & 0.95 & $0.5 L_{p, i}^{*}$ & 0.1 & 10 & 0 & 1 \\
\hline Max & 0.5 & 0.5 & 25 & 1.05 & $1.5 L_{p, i}^{*}$ & 0.999 & $10^{3}$ & 0.99 & 100 \\
\hline
\end{tabular}

The numerical signal obtained on a single layer of material $M_{1}$ is shown in Fig. 3 to give an idea of the noise level that has been added.
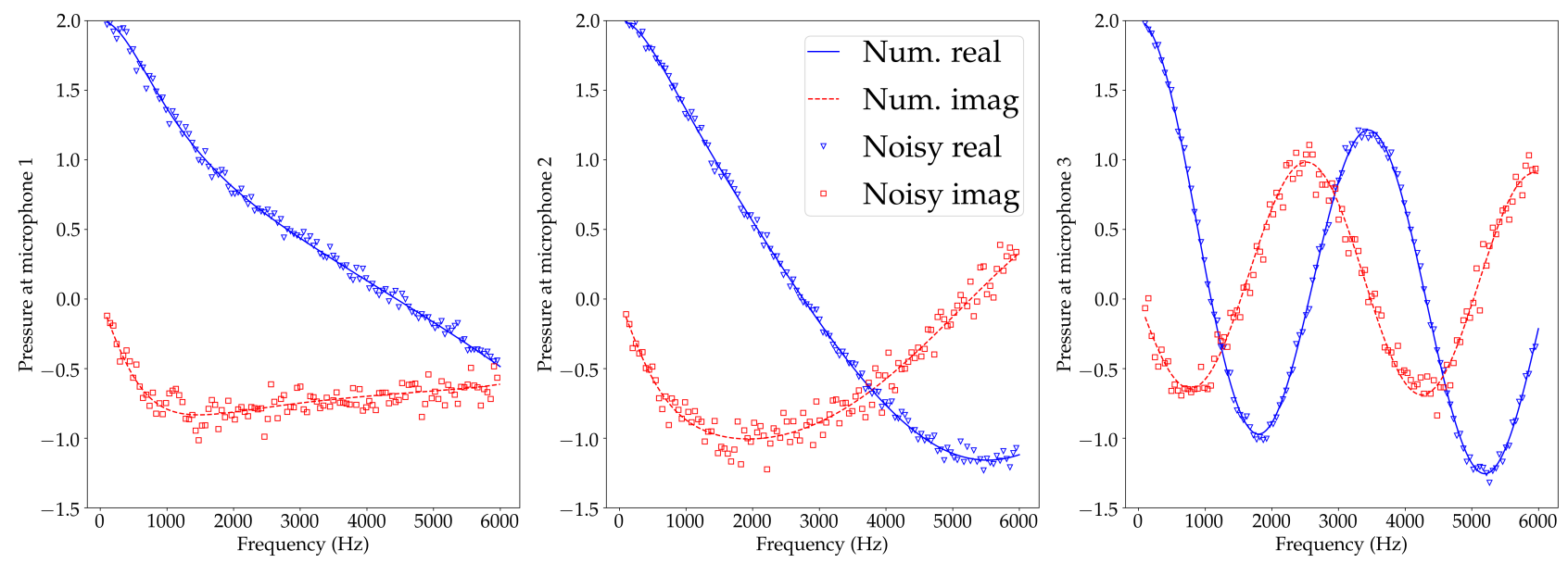

Figure 3: Microphone signals in the impedance tube for material $M_{1}$ at 3 microphone locations. 
The main take-away of the present work is to add additional observations on a multi-layer sample, via air-gaps placed behind the material. The presence of an air-gap changes the sample reflection coefficient, which can improve parameter estimation. This was attempted previously in Ref. [20], albeit with additional boundary conditions at the end of the impedance tube, and only for single layer materials.

Three different multi-layers are considered (among the 12 that were studied), with different configurations of samples $M_{1}, M_{2}$ and $M_{3}$. For all three multi-layers, two parameter estimations are performed. The first one, named "Case 1" in the figures, represents the standard default Bayesian inference, where only a single signal is used to perform the parameter estimation. The frequency range of the measured signal within the tube is, for this numerical test, [100-6000] Hz, with a step of about $1.2 \mathrm{~Hz}$, totalling 4800 data points. When adding observations with an air-gap, the total number of data points should increase. However, to keep a fair comparison between both parameter estimations, the signals are down-sampled so as to keep 4800 data points even when additional air-gaps are added to the Bayesian inference. In the second Bayesian inference, named "Case 2", there are 3 different air-gaps that have been added behind the sample (numerically) in addition to the no air-gap case. The maximum sample size reaches $9 \mathrm{~cm}$, which is close to the current limitation we have in our impedance tube sample holder.

We first display in Fig. 4 the identification results for parameters that do not pertain to the porous sample microstructure, i.e. the temperature, pressure and the noise standard deviations $\sigma_{\Re}$ and $\sigma_{\mathfrak{J}}$. While the pressure does not seem to easily identifiable, the remain parameters are quite well identified. This result held true in all the tests that were performed (bi- and tri-layers alike, totalling 12 different material assemblies).

We then display in Figs.5,6,7 the identification results pertaining to the samples microstructures.

Findings The main finding of the present work is that for the same number of observation, the Bayesian inference containing observations including the presence of an air-gap behind the material ("Case 2") systematically perform better than the reference case where no air-gap is included ("Case 1"). This is seen in Figs.5,6,7, where the pdf of "Case 2" are consistently closer to the true value, and more peaked. This peaked nature indicates that the information contained within the input data brings more information that in a case where the pdf would be broad. Also, the multi-modality of the pdfs, largely present in the reference case (see Fig. 7), has disappeared with the introduction of the data obtained with air-gaps.

\section{CONCLUSIONS}

It was first shown that using the surface impedance or reflection coefficient signals as the input of an inverse problem for the identification of the intrinsic properties of porous media would necessarily lead to biased parameter estimations. This is caused by an unknown frequency dependency of the uncertainty on these signals, which are derived initially from microphone pressure measurements using a nonlinear relationship. A more robust approach consists in directly using the pressure measurements at all the available microphones for the inverse problem input.

An objective Bayesian inference strategy was conducted on 3 different multi-layer assembly with 3 layers, to retrieve each layer's intrinsic properties, consisting in the parameters of Horoshenkov [17] applied to the JCAPL equivalent fluid model.

It was shown that adding multiple air-gaps behind a material to perform more additional acoustic observations could lead to an improved parameter estimation, removing the problem ill-posedness that can be present in the reference case without air-gap. This was shown in the case where the total amount of data was kept constant. 

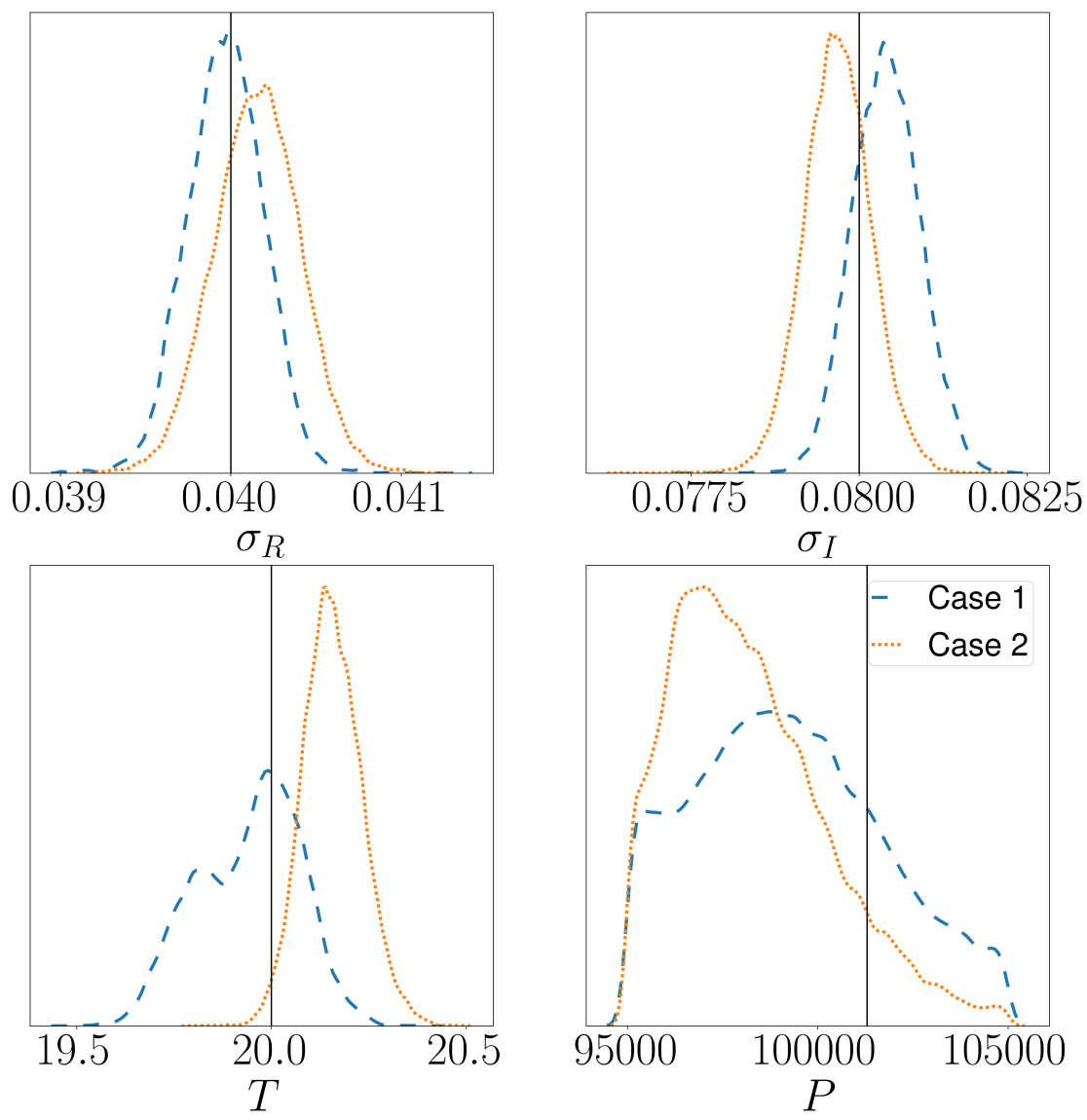

Figure 4: Probability density functions for the noise standard deviations $\sigma_{\Re}$ and $\sigma_{\Im}$, the temperature $\mathrm{T}$ and the ambient pressure $\mathrm{P}$. Units are the same as in 2. A vertical bar shows the true value. Case 1 is the reference case without any air gap, while Case 2 is the identification results with 3 air-gaps and the reference no air-gap case. Both inferences are conducted on the same amount of data. The multi-layer material is made of the serial assembly of $M_{1}, M_{2}$ and $M_{3}$ (from left to right in Fig. 1 .

\section{ACKNOWLEDGEMENTS}

We gratefully acknowledge Estelle Piot for her comments on an earlier version of this work. 

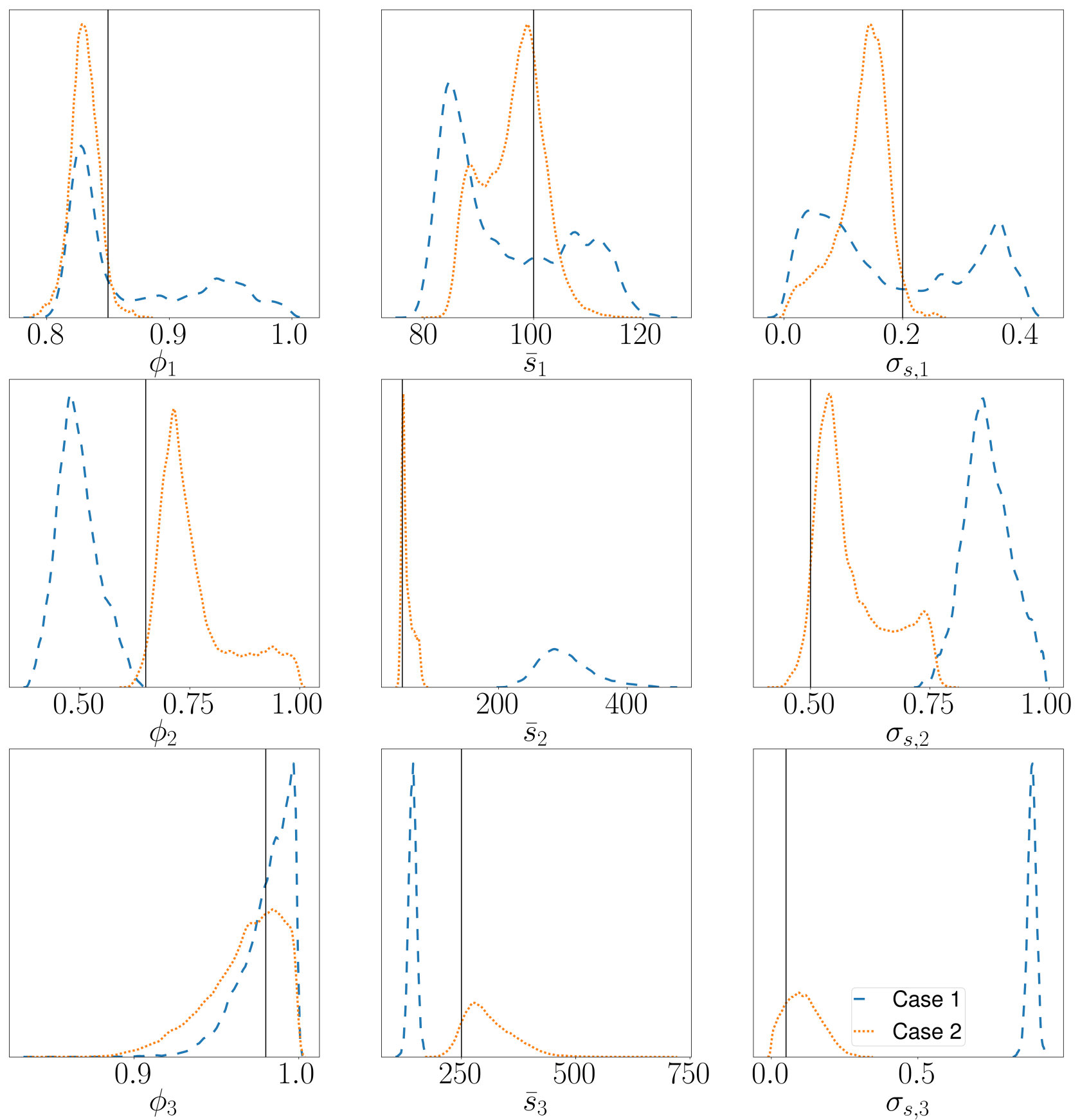

Figure 5: Probability density functions for the porosity $\phi$, the mean pore size $\bar{s}$ and the pore standard deviation $\sigma_{s}$. Units are the same as in 2. Case 1 is the reference case without any air gap, while Case 2 is the identification results with 3 air-gaps and the reference no air-gap case. Both inferences are conducted on the same amount of data. The multi-layer material is made of the serial assembly of $M_{1}, M_{2}$ and $M_{3}$ (from left to right in Fig. 1). 

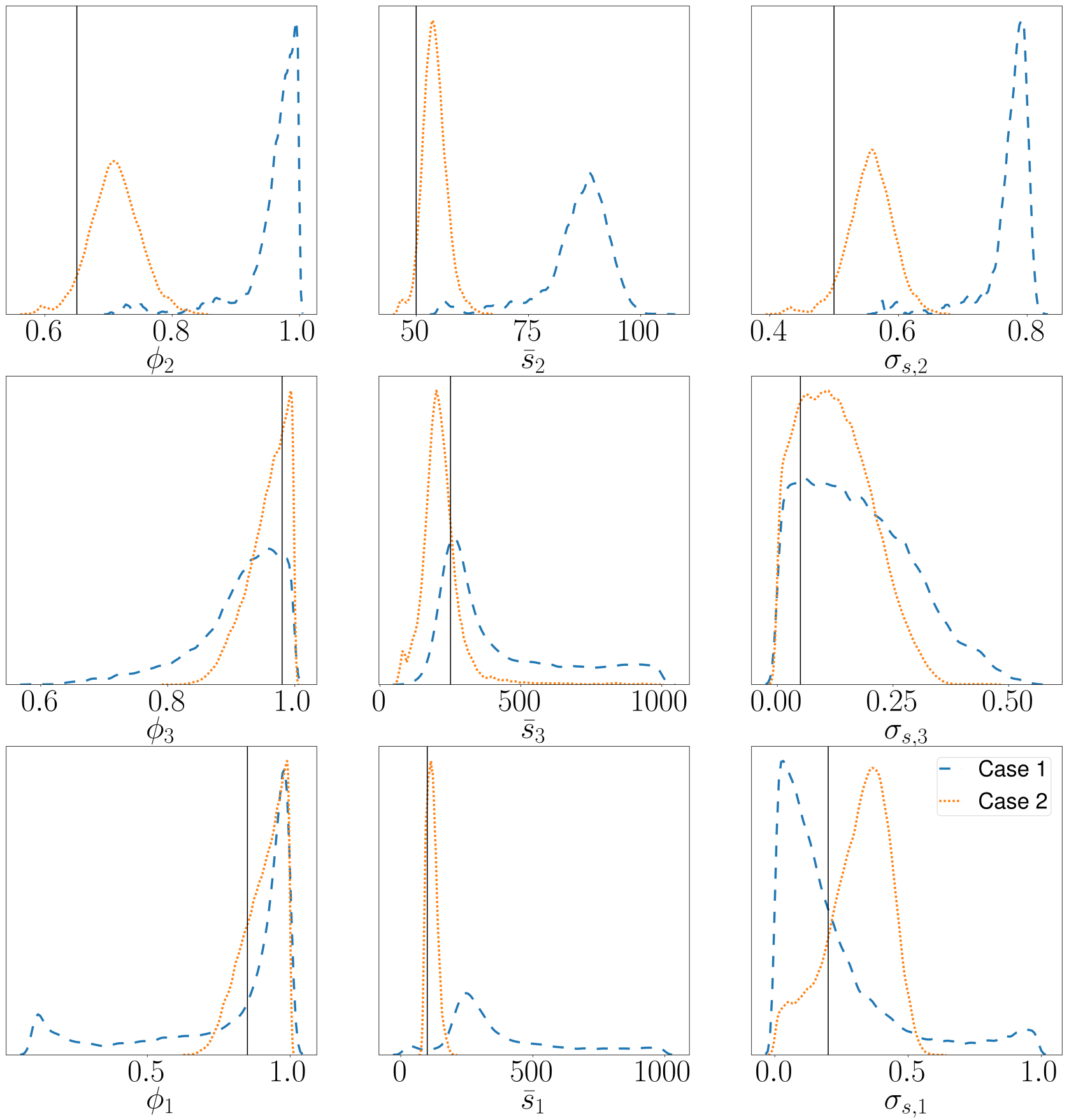

Figure 6: Probability density functions for the noise standard deviations $\sigma_{\mathfrak{R}}$ and $\sigma_{\mathfrak{I}}$, the temperature $\mathrm{T}$ and the ambient pressure $\mathrm{P}$. Units are the same as in 2 . Case 1 is the reference case without any air gap, while Case 2 is the identification results with 3 air-gaps and the reference no air-gap case. Both inferences are conducted on the same amount of data. The multi-layer material is made of the serial assembly of $M_{2}, M_{3}$ and $M_{1}$ (from left to right in Fig. 1). 

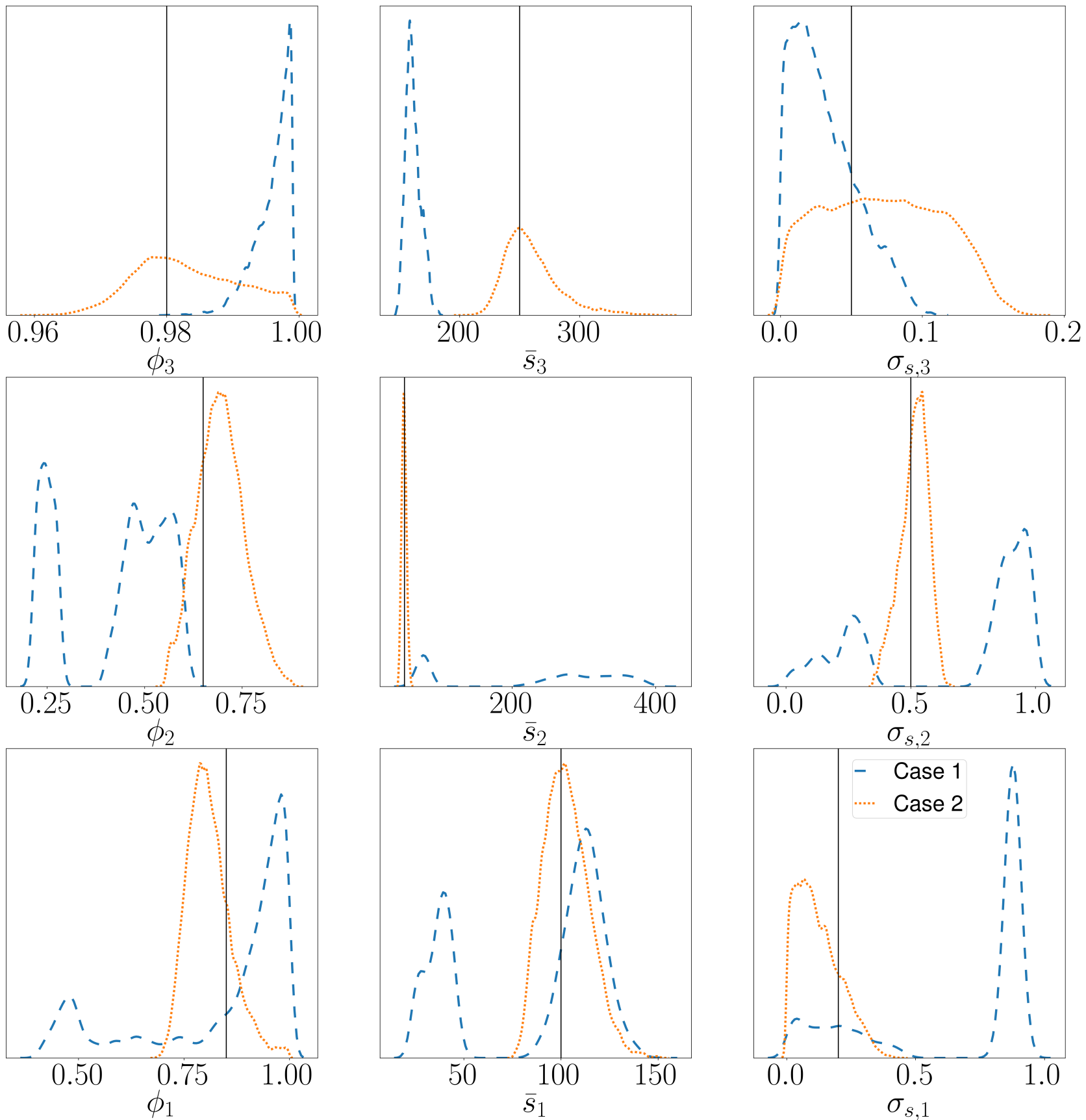

Figure 7: Probability density functions for the noise standard deviations $\sigma_{\Re}$ and $\sigma_{\Im}$, the temperature $\mathrm{T}$ and the ambient pressure $\mathrm{P}$. Units are the same as in 2 . Case 1 is the reference case without any air gap, while Case 2 is the identification results with 3 air-gaps and the reference no air-gap case. Both inferences are conducted on the same amount of data. The multi-layer material is made of the serial assembly of $M_{3}, M_{2}$ and $M_{1}$ (from left to right in Fig. 1). 


\section{REFERENCES}

[1] Youssef Atalla and Raymond Panneton. Inverse acoustical characterization of open cell porous media using impedance tube measurements. Canadian Acoustics, 33(1):11-24, 2005.

[2] Jean-Daniel Chazot, Erliang Zhang, and Jérôme Antoni. Acoustical and mechanical characterization of poroelastic materials using a bayesian approach. J. Acoust. Soc. Am., 131(6):4584-4595, 2012.

[3] Taissir Hentati, Leila Bouazizi, Mohamed Taktak, Hassen Trabelsi, and Mohamed Haddar. Multi-levels inverse identification of physical parameters of porous materials. J. Appl. Acoust., 2015.

[4] Tomasz G Zieliński. Normalized inverse characterization of sound absorbing rigid porous media. J. Acoust. Soc. Am., 137(6):3232-3243, 2015.

[5] M Niskanen, J-P Groby, A Duclos, O Dazel, JC Le Roux, N Poulain, T Huttunen, and T Lähivaara. Deterministic and statistical characterization of rigid frame porous materials from impedance tube measurements. J. Acoust. Soc. Am., 142(4):2407-2418, 2017.

[6] Cameron J. Fackler, Ning Xiang, and Kirill V. Horoshenkov. Bayesian acoustic analysis of multilayer porous media. The Journal of the Acoustical Society of America, 144(6):3582-3592, 2018.

[7] Kirill V Horoshenkov. A review of acoustical methods for porous material characterisation. Int. J. Acoust. Vib, 22:92-103, 2017.

[8] Laurent De Ryck, Walter Lauriks, Philippe Leclaire, Jean-Philippe Groby, Armand Wirgin, and Claude Depollier. Reconstruction of material properties profiles in one-dimensional macroscopically inhomogeneous rigid frame porous media in the frequency domain. The Journal of the Acoustical Society of America, 124(3):1591-1606, 2008.

[9] R. Roncen, Z. E. A. Fellah, D. Lafarge, E. Piot, F. Simon, E. Ogam, M. Fellah, and C. Depollier. Acoustical modeling and bayesian inference for rigid porous media in the low-mid frequency regime. The Journal of the Acoustical Society of America, 144(6):3084-3101, 2018.

[10] R Roncen, ZEA Fellah, E Piot, and E Ogam. Bayesian inference of a human bone and biomaterials using ultrasonic transmitted signals. The Journal of the Acoustical Society of America, 146(3):1629-1640, 2019.

[11] Rémi Roncen, Zine El Abiddine Fellah, and Erick Ogam. Improvements for the inverse acoustic characterization of multi-layer porous materials in impedance tube. 2021.

[12] Jean Allard and Noureddine Atalla. Propagation of Sound in Porous Media: Modelling Sound Absorbing Materials 2e. John Wiley \& Sons, New York, 2009.

[13] David Linton Johnson, Joel Koplik, and Roger Dashen. Theory of dynamic permeability and tortuosity in fluid-saturated porous media. J. Fluid Mech., 176:379-402, 1987.

[14] Yvan Champoux and Jean-F Allard. Dynamic tortuosity and bulk modulus in air-saturated porous media. J. Appl. Acoust., 70(4):1975-1979, 1991.

[15] Steven R Pride, Frank Dale Morgan, and Anthony F Gangi. Drag forces of porous-medium acoustics. Phys. Rev. B, 47(9):4964-4978, 1993.

[16] Denis Lafarge, Pavel Lemarinier, Jean F Allard, and Viggo Tarnow. Dynamic compressibility of air in porous structures at audible frequencies. J. Acoust. Soc. Am., 102(4):1995-2006, 1997.

[17] Kirill V Horoshenkov, Alistair Hurrell, and Jean-Philippe Groby. A three-parameter analytical model for the acoustical properties of porous media. The Journal of the Acoustical Society of America, 145(4):2512-2517, 2019.

[18] Ralph C Smith. Uncertainty quantification: theory, implementation, and applications, volume 12. Siam, Philadelphia, 2013. 
[19] Eric Laloy and Jasper A. Vrugt. High-dimensional posterior exploration of hydrologic models using multiple-try dream(zs) and high-performance computing. Water Resour. Res., 48(1):n/an/a, 2012. W01526.

[20] Nadine Sellen, Marie-Annick Galland, and Olivier Hilbrunner. Identification of the characteristic parameters of porous media using active control. In 8th AIAA/CEAS Aeroacoustics Conference, Breckenridge, Colorado, 2002. 